Monatsschr Kinderheilkd 2010 • 158:370-371

DOI 10.1007/s00112-010-2201-9

Online publiziert: 19. März 2010

(c) Springer-Verlag 2010

Redaktion

W. Rascher, Erlangen

H.W. Seyberth, London

\author{
J. Boos · Kommission für Arzneimittelsicherheit im Kindesalter (KASK) der \\ Deutschen Gesellschaft für Kinder- und Jugendmedizin (DGKJ) \\ Pädiatrische Hämatologie und Onkologie, Klinik und Poliklinik für Kinder \\ und Jugendmedizin, Westfälische Wilhelms-Universität Münster
}

\section{Sicherheit gibt es nicht umsonst ... oder doch?}

- Seit Jahrzehnten beklagen wir Lücken in der Arzneimittelversorgung für Kinder und dass besonders Kinder von Arzneimittelrisiken bedroht sind.

- Seit Jahrzehnten tragen Kinder diese Risiken und nehmen Kinderärzte in der Off-Label-Verschreibung erhebliche Teile der Verantwortung notgedrungen auf sich.

- Seit Jahrzehnten bleibt es der Kinderheilkunde überlassen, wesentliche Neuerungen im Arzneimittelbereich auf ihre Sinnhaftigkeit für Kinder und ihre komplexen Gesundheitsprobleme zu hinterfragen.

Seit 3 Jahren liegt nun eine europäische Kinderarzneimittelverordnung vor. Und spätestens seit diesem Winter wissen wir, dass sich damit vielleicht gar nicht so viel geändert hat. Natürlich werden pharmazeutische Unternehmen heute gezwungen, für ihre angestrebten Erwachsenenindikationen auch den Bedarf von Kindern darzulegen und entsprechende Studien durchzuführen - aber eben nur für die Erwachsenenindikationen. Alle Krankheitsbilder, die nur Kinder betreffen oder auch für Erwachsene nicht primär interessieren, bleiben unberücksichtigt. Die Arzneimittelentwicklung für Kinder wird zwar wirtschaftlich gestützt, bleibt aber freiwilligen Entscheidungen der pharmazeutischen Unternehmen überlassen. $\mathrm{Da}$ aber Arzneimittel mit dem Ziel des schnellen Marktzuganges zunächst in der Regel nur schmal für wenige, wirtschaftlich interessante Indikationen entwickelt werden, entsteht gerade für Kinder mit jeder Innovation schnell ein weites Feld verpasster Möglichkeiten - ein Feld für eige- ne Studien, Heilversuche und Off-LabelEinsätze.

Selbst bei den für Kinder durchgeführten Prüfungen entgehen weder Behörden noch Unternehmen dem Problem, dass in Studien für Kinder in der Regel viel geringere Patientenzahlen rekrutiert werden können als bei Erwachsenen.

\section{( Kompromisse bei der Zulassung neuer Medikamente für Kinder sind unumgänglich}

Daraus resultieren auch eine geringere statistische Sicherheit und weniger zwingende Evidenz. Gleiche Evidenz und Sicherheitsdaten hätten lange Entwicklungszeiten mit langwierigen Phase-IIIStudien zur Voraussetzung und eine erheblich verzögerte Zulassung neuer Medikamente für Kinder zur Folge. Kompromisse sind also erforderlich.

Es stehen somit öfter Arzneiformen, die auch Kinder einnehmen können, zur Verfügung, vielleicht gibt es endlich auch klarere Dosierungsvorgaben, wodurch eine Reihe von Fehlerquellen ausgeschaltet werden können. Das stellt zwar einen Fortschritt dar, aber wirkliche Arzneimittelsicherheit erhalten wir auf diese Weise nicht.

Es war nicht überraschend, dass auch kleine Kinder von Grippeviren bedroht sind. Es war nicht überraschend, dass TNF auch in der Kinderheilkunde Hoffnungen und Erwartungen weckt und neue Optionen (und Indikationen) eröffnet. Und doch bestehen im ersten Fall weiterhin ernst zu nehmende Versorgungs- und Informationslücken z. B. mit Oseltami$\operatorname{vir}\left(\mathrm{Tamilfu}^{\circledR}\right)$. Es waren einzelne Kinder- kliniken, die die ersten Sicherheitsdaten für diese Arznei bei Säuglingen zusammentrugen [4], die Firma Hoffmann-La Roche hielt sich bei Säuglingen und Kleinkindern eher zurück, hatte jedenfalls erkennbar keine Eile. Die aktuelle Zulassung durch die EMEA (European Medicines Agency) für Kinder unter 12 Monaten und besonders die Empfehlungen für Kinder im ersten Lebensmonat wirken eher der Not geschuldet als durch überzeugende Daten begründet [2].

Und im Falle des Tumornekrosefaktorinhibitors wird soeben deutlich, dass auch die spezifische Manipulation biologischer Systeme nicht ohne Risiken ist. Die Sicherheit der Hemmung des Tumornekrosefaktors gerade in Bezug auf Malignomentstehung scheint nun (nicht ganz überraschend) fraglich [3].

Wenn wir zumindest für unheilbar, schwer und chronisch kranke Kinder aber früh und mit anwendbaren Präparaten Zugang zu neuen Therapieoptionen erhalten wollen, wenn wir dabei die $\mathrm{Zu}$ lassungsbehörden zu konstruktiven Kompromissen auffordern, dann müssen wir als Kinderärzte im gesamten Prozess kooperieren und alles Mögliche zur frühen Entdeckung von Sicherheitsrisiken und unerwarteten Nebenwirkungen beitragen. Nur klare Risikobegrenzung durch systematische Beobachtung auch nach der Zulassung durch Risikomanagementprogramme der verantwortlichen Unternehmen und konsequente Beteiligung aller verordnenden Ärzte am Spontanmeldesystem können hier einen Ausweg bieten. Wirkungen und Wirksamkeit lassen sich meist relativ schnell erfahren. Risiken und Nutzen-Risiko-Bewertungen erfordern ei- 
ne kontinuierlich kritische Begleitung der Verordnungen auch außerhalb von Studien und gerade nach der Zulassung.

Der oft diskutierte Aufbau eines pädiatrischen Pharmakovigilanzzentrums stößt seit langem auf verschiedenste Schwierigkeiten, die hier nicht erörtert werden können. So etwas gibt es nicht umsonst, und offensichtlich ist an dieser Stelle auch die Zuständigkeit für die Finanzierung unklar, es stellt sich die Frage, ob Arzneimittelsicherheit eher eine Frage der Forschung oder der Versorgung ist.

\section{Ist Arzneimittelsicherheit eher eine Frage der Forschung oder der Versorgung?}

Eindeutig ist aber die Verantwortung der pharmazeutischen Unternehmen für ihre Präparate und die der Behörden für die Sicherheit im Verkehr mit Arzneimitteln. Die Instrumente für pädiatrische Arzneimittelsicherheit sind vielfältig und in Leitlinien diskutiert [1]. Auflagen in Bezug auf Phase-IV-Studien können von Behörden verordnet werden, die Firmen müssen Risikomanagementpläne vorlegen und regelmäßige Sicherheitsberichte abliefern. Das alles geht mit finanziellen Aufwendungen einher, aber die wirtschaftlichen Ressourcen sind nicht zuletzt durch die EU-Kinderarzneimittelverordnung eindeutig vorhanden.

Die Kontrolle der Anwendungen, die Beobachtungen am Kind und damit die Sammlung der Quelldaten aber sind ausschließlich den verordnenden Ärzten/-innen möglich und (fast) umsonst zu erbringen:

- Melden Sie UAW (unerwünschte

Arzneimittelwirkungen).

- Benennen Sie einen Mitarbeiter/-in für Arzneimittelsicherheit - manche Frage kann schon bei der Anmeldung gestellt werden, und etwas Fortbildung macht Spaß.

- Fragen Sie ihren Pharmareferenten regelmäßig nach speziell für Kinder initiierten Risikomanagementinitiativen.

- Lassen sie sich auch einmal die Sicherheitsberichte zeigen.

- Fragen Sie selbst oder über ihre Fachgesellschaften bei den Behörden re- gelmäßig zu den Ihnen wichtigen Arzneistoffen an.

Ein wirkliches Interesse, regelmäßiges Thematisieren von Arzneimittelsicherheit, ein Umdenken auch im eigenen Verantwortungsbereich - das alles kostet nichts, wird aber bei Behörden und auch der pharmazeutischen Industrie Aktivitäten für altersbezogene Auswertungen und evtl. auch den Aufbau von sinnvollen Strukturen in Gang setzen.

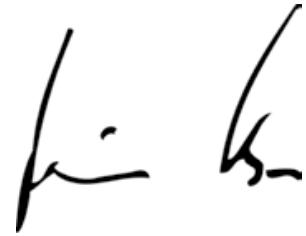

Prof. Dr. J. Boos

\section{Korrespondenzadresse}

\section{Prof. Dr. J. Boos}

für die Kommission für Arzneimittelsicherheit im Kindesalter (KASK) der Deutschen Gesellschaft für Kinder- und Jugendmedizin (DGKJ) Pädiatrische Hämatologie und Onkologie, Klinik und Poliklinik für Kinder und Jugendmedizin, Westfälische Wilhelms-Universität Münster Albert-Schweitzer-Straße 33, 48149 Münster boosj@uni-muenster.de

\section{Literatur}

1. EMEA (2007) Guideline on conduct of pharmacovigilance for medicines used by the paediatric population. EMEA, London, 25.01.2007, EMEA/CHMP/ PhVWP/235910/2005 - rev 1

2. EMEA (2010) ANNEX I - summary of product characteristics. EMEA, London, www.ema.europa.eu/ humandocs/PDFs/EPAR/tamiflu/emea-combinedh402en-21Jan10.pdf

3. Horneff G, Hospach C, Dannecker G et al (2010) Juvenile idiopathische Arthritis - Therapie mit TNFaInhibitoren. Monatsschr Kinderheilkd 3

4. Siedler K, Skopnik H (2010) Oseltamivir for treatment of influenza in infants less than one year: a retrospective analysis. Pediatr Infect Dis J Feb 10. [Epub ahead of print]

\section{Ausschreibung: Nachsorgepreis der Deutschen Kinderkrebs- nachsorge}

Die Deutsche Kinderkrebsnachsorge-Stiftung für das chronisch kranke Kind - vergibt für zukunftsweisende Arbeiten, Projekte und Initiativen der stationären und ambulanten familienorientierten Nachsorge und Betreuung den Nachsorgepreis der Deutschen Kinderkrebsnachsorge-Stiftung für das chronisch kranke Kind.

Der Förderpreis ist mit 10.000 Euro dotiert. Bewerbungen können für alle Projekte der Nachsorge chronisch kranker Kinder und deren Familien eingereicht werden.

Für die Vergabe des Nachsorgepreises sind Bestimmungen maßgebend, die bei der Deutschen Kinderkrebsnachsorge angefordert werden können.

Die Verleihung des Preises erfolgt im November 2010.

Bewerbungsfrist: 31. Juli 2010

Kontakt:

Deutsche Kinderkrebsnachsorge-Stiftung für das chronisch kranke Kind

Gemeindewaldstr. 75

78052 VS-Tannheim

Tel. 07705 - 920500

Fax 07705 - 920191

Mail: info@kinderkrebsnachsorge.de 